\title{
Efeitos da adição de propilenoglicol ou monensina à silagem de milho sobre a cinética de degradação dos carboidratos e produção cumulativa de gases in vitro
}

\author{
[Effects of adding propylene glycol or monensin to corn silage on the degradation kinetics of \\ carbohydrates and in vitro cumulative gas production] \\ B.N. Faria ${ }^{1}$, R.B. Reis ${ }^{2}$, R.M. Mauricio ${ }^{3}$, A.M.Q. Lana' ${ }^{2}$, S.R.V. Soares ${ }^{1}$, \\ H.M. Saturnino ${ }^{2}$ S.G. Coelho ${ }^{2}$ \\ ${ }^{1}$ Aluno de pós-graduação - EV-UFMG - Belo Horizonte, MG \\ ${ }^{2}$ Escola de Veterinária - UFMG - Belo Horizonte, MG \\ ${ }^{3}$ Fundação Ezequiel Dias - Belo Horizonte, MG
}

\begin{abstract}
RESUMO
Avaliaram-se os efeitos dos aditivos propilenoglicol e/ou monensina sobre a degradabilidade média e efetiva dos carboidratos totais, $\mathrm{pH}$ e produção cumulativa de gases da silagem de milho por meio da técnica in vitro semi-automática de produção de gases. Os tratamentos constituíram-se de silagem de milho (SM); SM associada ao propilenoglicol (SM+PG); $\mathrm{SM}$ associada à monensina (SM+MO); $\mathrm{SM}$ associada ao propilenoglicol e à monensina $(\mathrm{SM}+\mathrm{PG}+\mathrm{MO})$ avaliados com duas, quatro, seis, 12, 24, 48 e 96 horas. A adição de monensina ou monensina associada ao propilenoglicol aumentou $(\mathrm{P}<0,05)$ a degradabilidade dos carboidratos totais às duas horas. $\mathrm{SM}+\mathrm{MO}$ apresentou maior degradabilidade efetiva dos carboidratos totais em todas as taxas de passagem. A utilização de monensina reduziu a produção cumulativa de gases das 12 às 96 horas. Entre os tratamentos, $\mathrm{SM}+\mathrm{MO}$ apresentou o menor potencial de produção de gases $(221 \mathrm{ml} / \mathrm{g}$ carboidratos totais) e o menor tempo de colonização (1,08 horas) em relação aos tratamentos SM e SM+PG (1,58 e 1,49 horas, respectivamente). A produção cumulativa de gases e degradabilidade dos carboidratos totais apresentaram elevada correlação, variando de 94 a $97 \%(\mathrm{P}<0,01)$. O pH do meio foi inversamente correlacionado à degradabilidade dos carboidratos totais $(\mathrm{r}=-79 \%, \mathrm{P}<0,01)$. $\mathrm{O}$ uso de monensina pode ser uma boa alternativa para se melhorarem os parâmetros da cinética de degradação da silagem de milho.
\end{abstract}

Palavras-chave: silagem de milho, aditivos, degradação efetiva, ionóforos, produção cumulativa de gases

\begin{abstract}
The effects of the additives propylene glycol and/or monensin on the degradation of total carbohydrates, $p H$, and cumulative gas production of corn silage by the semi-automated in vitro gas production technique were evaluated. The treatments were corn silage (CS); CS plus propylene glycol $(C S+P G)$; CS plus monensin $(C S+M O)$, and $C S$ plus propylene glycol and monensin $(C S+P G+M O)$, which were evaluated at two, four, six, 12, 24, 48, and 96 hours. The addition of monensin or monensin plus propylene glycol increased $(P<0.05)$ the degradation of total carbohydrates at $2 \mathrm{~h}$. The effective degradations of total carbohydrates for $C S+M O$ treatment (55.2; 42.7; and 36.5\%) were the highest in all passage rates. The use of monensin reduced cumulative gas production from 12 to $96 \mathrm{~h}$. CS+MO treatment had the lowest potential of gas production $(221 \mathrm{ml} / \mathrm{g}$ total carbohydrates), and the lowest Lag phase (1.08h), as compared to CS and CS+PG treatment (1.58 and 1.49h, respectively). Cumulative gas production and degradation of total carbohydrates were highly correlated (94 to 97\%; $P<0.01)$. The $p H$ was inversely correlated to degradability of total carbohydrates $(r=-$ 0.79; $P<0.01$ ). Thus, monensin may be used for improving the ruminal degradability of corn silage.
\end{abstract}

Keywords: corn silage, additives, effective degradation, ionophor, cumulative gas production

Recebido em 1 de julho de 2007

Aceito em 30 de maio de 2008

*Autor para correspondência (corresponding author)

E-mail: rbreis@vet.ufmg.br 


\section{INTRODUÇÃO}

A utilização de aditivos na dieta de ruminantes é uma prática cada vez mais comum na bovinocultura moderna. Dentre os aditivos, destacam-se os ionóforos e o propilenoglicol, duas substâncias que têm como função aumentar a disponibilidade de energia para o animal (Faria et al., 2008).

Os ionóforos são antibióticos coccidiostáticos que exercem ação seletiva sobre bactérias Gram positivo. Quando utilizados na dieta de ruminantes, atuam sobre a microbiota ruminal, promovendo a queda na população dessas bactérias, e têm como efeitos principais o aumento na digestibilidade da matéria seca (MS), a redução da produção de calor e o aumento da energia líquida de mantença da dieta. Além disso, promovem efeito protetor sobre a proteína da dieta e reduzem a produção de ácido lático (Goodrich et al., 1984). Dentre os vários ionóforos conhecidos, cerca de 120 formas naturais (Nagaraja et al., 1997), os mais utilizados na alimentação de bovinos são a monensina e a lasalocida.

O propilenoglicol é um diálcool utilizado em vários alimentos processados como umectante, agente plastificante, conservante e, quando em grandes quantidades, como substrato para a síntese de carboidratos nos animais. Quando adicionado à dieta, o propilenoglicol pode ser utilizado pela microbiota ruminal promovendo alteração da proporção e aumento da produção de ácidos graxos voláteis (AGV) (Grummer et al., 1994). Pouco se sabe, no entanto, sobre sua influência na degradação dos alimentos no rúmen.

Este trabalho teve o objetivo de avaliar o uso de aditivos propilenoglicol e/ou monensina sobre a cinética de degradação, degradação efetiva e degradação dos carboidratos totais da silagem de milho por meio da técnica in vitro semiautomática de produção de gases.

\section{MATERIAL E MÉTODOS}

A silagem de milho utilizada apresentou 25,6\% de matéria seca (MS); $8,4 \%$ de proteína bruta (PB); $54,9 \%$ de fibra em detergente neutro (FDN) e $27,4 \%$ de fibra em detergente ácido
(FDA). Como aditivos, foram utilizados a monensina (MO, $100 \%$ de pureza) e o propilenoglicol (PG, 99\% de pureza).

Os substratos constituíram da incubação da silagem de milho sem aditivo (SM); da SM associada ao propilenoglicol ( $\mathrm{SM}+\mathrm{PG})$; da $\mathrm{SM}$ associada à monensina $(\mathrm{SM}+\mathrm{MO})$ e, da $\mathrm{SM}$ associada ao propilenoglicol e à monensina $(\mathrm{SM}+\mathrm{PG}+\mathrm{MO})$. Para cada tratamento, foram utilizados 21 frascos de fermentação, sendo três frascos para cada tempo de avaliação. Outros 84 frascos foram utilizados com a finalidade de medir a produção de gases na ausência do substrato em cada tempo de avaliação, utilizados para a correção dos resultados, sendo três sem silagem de milho e aditivos, três com propilenoglicol, três com monensina e três com propilenoglicol e monensina.

O cálculo da quantidade de aditivo utilizado em cada frasco foi feito com base na ingestão diária de matéria seca da doadora do conteúdo ruminal. Foram consideradas as doses de $300 \mathrm{mg}$ e $300 \mathrm{ml}$ por animal/dia para a monensina e propilenoglicol, respectivamente. A partir desses cálculos foram utilizados $0,27 \mathrm{mg}$ de monensina $\mathrm{e}$ $0,013 \mathrm{ml}$ de propilenoglicol em cada frasco de fermentação contendo $1 \mathrm{~g}$ de matéria seca (MS) do substrato. Os aditivos foram diluídos em solvente inerte (dimetilsulfóxido) e adicionados com o auxílio de micropipeta de $1000 \mu$ l.

Os parâmetros cinéticos de degradação ruminal foram analisados por meio da técnica in vitro semi-automática de produção de gases (TIVSAPG) (Maurício et al., 1999). O inóculo foi preparado com líquido ruminal obtido de uma vaca da raça Holandesa, em lactação, mediante uso de cânula ruminal. $\mathrm{O}$ animal estava adaptado à dieta à base de silagem de milho, farelo de soja, milho triturado e polpa cítrica, com $18 \%$ de PB e relação concentrado/volumoso de 46/56, com base na MS, fornecida na forma de ração total. $\mathrm{O}$ conteúdo ruminal foi retirado antes do fornecimento da dieta e armazenado em garrafas térmicas previamente aquecidas e imediatamente transportadas para o laboratório de nutrição animal.

As leituras de pressão foram realizadas às duas, quatro, seis, oito, 10, 12, 15, 19, 24, 30, 36, 48, 72 e 96 horas após a inoculação. Os dados de 
pressão foram utilizados para o cálculo do volume de gases produzidos de acordo com Mauricio et al. (2003) e Nogueira et al. (2006).

Após a leitura da pressão, realizada às duas, quatro, seis, 12, 24, 48 e 96 horas de fermentação, três frascos de fermentação (triplicata) foram retirados de cada tratamento. Os resíduos de fermentação foram obtidos por meio de filtragem do material contido nos frascos de fermentação em cadinhos de porosidade de um mícron ${ }^{1}$ acrescidos de lã de vidro.

Os resíduos de fermentação ainda foram secos por 24 horas em estufa a $100^{\circ} \mathrm{C}$ e pesados para cálculo dos valores de resíduo não degradado da matéria seca.

No momento da filtragem das amostras, por meio dos cadinhos de vidro, cerca de $15 \mathrm{ml}$ do resíduo líquido da fermentação foram coletados em béquer de $20 \mathrm{ml}$ e utilizados para a mensuração do $\mathrm{pH}$.

O delineamento estatístico para avaliar a degradabilidade da matéria seca, variações no $\mathrm{pH}$ do meio e produção de gases foi em blocos ao acaso no esquema de parcelas subdivididas, sendo a parcela composta por um fatorial $2 \times 2$ (com e sem MO e com e sem PG) e as subparcelas, os tempos de retirada dos frascos de fermentação (duas, quatro, seis, 12, 24, 48 e 96h).

Para verificar a normalidade e homocedasticidade de variâncias, utilizaram-se os testes de Lilliefors e Bartlett, respectivamente. O teste estatístico, utilizado na comparação de substratos, foi o de Fisher (teste F) $\operatorname{com} \alpha=0,05$. Para a degradação de carboidratos totais foi realizado o ajuste de modelo de regressão em função de tempos de avaliação. Os dados foram analisados pelo programa estatístico SAEG (Sistema...,1997).

O modelo de France et al. (1993) foi utilizado para descrever o potencial máximo de produção de gases (A), o tempo de colonização (L) e a taxa de produção de gases $(\mu)$ por meio do programa MLP (Official..., 1980).

${ }^{1}$ Pirex - Vidrotec Ltda. - São Paulo, Brasil.
Foram ainda calculadas as taxas de degradação efetiva dos carboidratos totais para as taxas de passagem de $2 \%, 5 \%$ e $8 \% / \mathrm{h}$ para baixo, médio e alto consumo, respectivamente, conforme especificações do AFRC (Nutritive..., 1992), empregando-se a equação proposta por France et al. (1993).

\section{RESULTADOS E DISCUSSÃO}

Os valores de degradabilidade média dos carboidratos totais são apresentados na Tab. 1. A utilização da monensina (SM+MO) e sua associação com o propilenoglicol (SM+PG+MO) propiciaram aumento $(\mathrm{P}<0,05) \quad$ na degradabilidade da silagem de milho nas duas primeiras horas de fermentação (19,3 e 18,9\%, respectivamente), quando comparado aos tratamentos SM (14,0\%) e SM+PG (12,9\%).

Às 96 horas de avaliação, a degradação foi maior na silagem de milho sem aditivo e na silagem de milho com monensina e propilenoglicol.

As taxas de degradação dos carboidratos de acordo com os modelos de regressão para a silagem de milho acrescida ou não de aditivos estão na Tab. 2.

A utilização de MO com ou sem PG resultou em degradação mais rápida da silagem de milho durante as primeiras seis horas de fermentação. A partir das primeiras seis horas, a velocidade de degradação alterou-se em função dos aditivos utilizados. Os valores de degradação dos carboidratos totais às 24 horas foram próximos, 48,$8 ; 47,7 ; 48,2$ e $47,7 \%$ para $\mathrm{SM}$, $\mathrm{SM}+\mathrm{PG}$, $\mathrm{SM}+\mathrm{MO}$ e $\mathrm{SM}+\mathrm{PG}+\mathrm{MO}$, respectivamente. Às 96 horas de fermentação, a taxa de degradação da silagem de milho sem aditivos foi de $85,2 \%$ e de 80,$8 ; 76,8$ e $76,2 \%$ para $\mathrm{SM}+\mathrm{PG}, \mathrm{SM}+\mathrm{MO}$ e $\mathrm{SM}+\mathrm{PG}+\mathrm{MO}$, respectivamente.

A adaptação ruminal à MO parece ser importante para os processos de utilização da matéria seca. Para Schelling (1984), o efeito da adaptação à monensina não tem sido estudado de forma organizada, mas vários trabalhos sugerem que a utilização de ionóforos pode produzir, inicialmente, um efeito negativo sobre a degradação da matéria seca. Simpson (1978) observou decréscimo na digestibilidade da celulose pela falta de um período de adaptação. Esses efeitos, porém, foram corrigidos com período de adaptação de 21 dias. 
Efeitos da adição de propilenoglicol...

Tabela 1. Degradabilidade média (\%) dos carboidratos totais da silagem de milho associada ou não ao propilenoglicol e/ou à monensina

\begin{tabular}{lccccccc}
\hline \multirow{2}{*}{ Substrato } & \multicolumn{7}{c}{ Tempo pós-inoculação (h) } \\
\cline { 2 - 7 } & 2 & 4 & 6 & 12 & 24 & 48 & 96 \\
\hline SM & $14,04 \mathrm{~b}$ & $27,41 \mathrm{a}$ & $28,68 \mathrm{a}$ & $46,72 \mathrm{a}$ & $47,42 \mathrm{a}$ & $59,78 \mathrm{a}$ & $79,93 \mathrm{a}$ \\
SM+PG & $12,92 \mathrm{~b}$ & $30,56 \mathrm{a}$ & $30,56 \mathrm{a}$ & $45,07 \mathrm{a}$ & $47,68 \mathrm{a}$ & $59,99 \mathrm{a}$ & $70,70 \mathrm{a}$ \\
SM+MO & $19,28 \mathrm{a}$ & $27,28 \mathrm{a}$ & $28,58 \mathrm{a}$ & $45,37 \mathrm{a}$ & $49,34 \mathrm{a}$ & $60,07 \mathrm{a}$ & $71,49 \mathrm{a}$ \\
SM+PG+MO & $18,88 \mathrm{a}$ & $26,13 \mathrm{a}$ & $27,83 \mathrm{a}$ & $46,59 \mathrm{a}$ & $49,99 \mathrm{a}$ & $61,57 \mathrm{a}$ & $67,20 \mathrm{~b}$ \\
\hline
\end{tabular}

SM: silagem de milho; PG: propilenoglicol; MO: monensina.

Médias seguidas de letras distintas na coluna diferem entre si pelo teste $\mathrm{F}(\mathrm{P}<0,05)$. CV: $2,6 \%$.

Tabela 2. Degradação média dos carboidratos totais (\%) da silagem de milho associada ou não ao propilenoglicol e/ou à monensina de acordo com os modelos de regressão

\begin{tabular}{|c|c|c|c|c|c|c|c|c|}
\hline \multirow{2}{*}{ Substrato } & \multicolumn{7}{|c|}{ Tempo pós-inoculação (h) } & \multirow{2}{*}{$r^{2}(\%)$} \\
\hline & 2 & 4 & 6 & 12 & 24 & 48 & 96 & \\
\hline SM & \multicolumn{7}{|c|}{$\log ($ degrad. $)=1,1319+0,4029 * \log ($ tempo $)$} & 91,23 \\
\hline $\mathrm{SM}+\mathrm{PG}$ & & \multicolumn{6}{|c|}{$\log ($ degrad. $)=1,1528+0,3808 * \log ($ tempo $)$} & 84,18 \\
\hline $\mathrm{SM}+\mathrm{MO}$ & & \multicolumn{6}{|c|}{$\log ($ degrad. $)=1,2198+0,3358 * \log ($ tempo $)$} & 95,59 \\
\hline $\mathrm{SM}+\mathrm{PG}+\mathrm{MO}$ & & \multicolumn{6}{|c|}{$\log ($ degrad. $)=1,2119+0,3380 * \log ($ tempo $)$} & 93,53 \\
\hline
\end{tabular}

SM: silagem de milho; PG: propilenoglicol; MO: monensina.

Neste experimento não houve adaptação prévia da doadora de líquido ruminal. Não obstante, a falta de adaptação não resultou em efeito negativo sobre a degradação dos carboidratos totais nas primeiras 48 horas de fermentação. É provável que isso tenha ocorrido devido às características da TIVSAPG, um sistema fechado, que mantém constante a concentração de monensina no meio durante todo o processo de fermentação, o que leva a maior pressão de seleção das bactérias ruminais. Essa maior pressão conduziria a mudanças mais rápidas e uniformes da microbiota ruminal, diminuindo os efeitos da falta de adaptação da doadora sobre a queda na degradação dos carboidratos totais.

$\mathrm{O}$ PG tem sido relacionado à maior produção de propionato ruminal. De acordo com Clapperton e Czerkawski (1972), o PG pode ser fermentado in vitro por microrganismos ruminais, ocorrendo acúmulo temporário de ácido lático e produção de ácido propiônico e acético sem o acúmulo adicional de metano. Segundo esses autores, as alterações estão relacionadas à transformação direta do aditivo pela microbiota ruminal. Apesar desses efeitos sobre a produção de ácidos graxos, pouco se sabe ou se tem pesquisado sobre a influência do PG na degradação ruminal. Neste experimento, a utilização desse aditivo, aparentemente, não alterou a degradação dos carboidratos totais da silagem de milho. A manutenção da degradação da silagem de milho e a maior produção de ácidos graxos voláteis promoveriam o maior aporte energético para o animal suplementado com dietas a base de silagem de milho e suplementadas com PG, possibilitando maiores índices de produção animal.

As degradabilidades efetivas dos carboidratos totais da silagem de milho adicionada ou não de propilenoglicol e/ou de monensina, calculadas para taxas de passagem de 0,$02 ; 0,05$ e $0,08 / \mathrm{h}$ são mostradas na Tab. 3 .

Tabela 3. Degradabilidades efetivas (\%) dos carboidratos totais da silagem de milho associada ou não ao propilenoglicol e/ou à monensina calculadas para taxas de passagem de 0,$02 ; 0,05$ e $0,08 / \mathrm{h}$

\begin{tabular}{lccc} 
& \multicolumn{3}{c}{ Taxa de passagem $(\% / \mathrm{h})$} \\
\cline { 2 - 4 } & 0,02 & 0,05 & 0,08 \\
\hline SM & 54,94 & 41,48 & 34,65 \\
SM+PG & 53,38 & 40,32 & 33,74 \\
SM+MO & 55,21 & 42,68 & 36,48 \\
SM+PG+MO & 51,03 & 39,28 & 33,14 \\
\hline
\end{tabular}

SM: silagem de milho, PG: propilenoglicol, MO: monensina. 
Apesar de o inóculo ter sido oriundo de animal não adaptado à monensina, não foram observados efeitos negativos do aditivo sobre a degradabilidade efetiva dos carboidratos totais. A utilização da monensina promoveu elevação da degradabildiade, sendo $4,9 \%$ mais alta que a do tratamento-controle, nas primeiras duas horas de fermentação.

A utilização de monensina $(\mathrm{SM}+\mathrm{MO})$ resultou em valores mais altos de degradabilidade efetiva dos carboidratos totais em todas as taxas de passagem em relação aos demais tratamentos. Já o uso de PG com ou sem MO resultou em valores mais baixos que os observados para a silagem de milho sem nenhum aditivo.

Os valores de produção cumulativa de gases e o potencial máximo de produção de gases, o tempo de colonização e a taxa de produção de gases, estimados pelo modelo de France et al. (1993) para a silagem de milho com ou sem os aditivos, encontram-se na Tab. 4.

Tabela 4. Produção cumulativa de gases e parâmetros de produção de gases estimados, pelo modelo de France et al. (1993), da silagem de milho associada ou não ao propilenoglicol e/ou à monensina

\begin{tabular}{|c|c|c|c|c|c|c|c|c|}
\hline \multirow[t]{2}{*}{ Tratamento } & \multicolumn{5}{|c|}{$\begin{array}{l}\text { Produção cumulativa de gases } \\
\text { (ml/g de carboidrato total) }\end{array}$} & \multirow[t]{2}{*}{ A } & \multirow[t]{2}{*}{ Lag } & \multirow[t]{2}{*}{$\mu$} \\
\hline & $6 \mathrm{~h}$ & $12 \mathrm{~h}$ & $24 \mathrm{~h}$ & $48 \mathrm{~h}$ & $96 \mathrm{~h}$ & & & \\
\hline SM & $48,99 a$ & $112,13 \mathrm{ab}$ & $174,40 \mathrm{a}$ & $221,09 a$ & $249,58 \mathrm{a}$ & 245 & 1,58 & 0,06 \\
\hline $\mathrm{SM}+\mathrm{PG}$ & $48,70 \mathrm{a}$ & $112,10 \mathrm{ab}$ & $176,33 a$ & $224,57 a$ & $250,88 \mathrm{a}$ & 246 & 1,49 & 0,05 \\
\hline $\mathrm{SM}+\mathrm{MO}$ & $46,72 \mathrm{a}$ & $104,44 b$ & $164,76 b$ & $207,00 \mathrm{~b}$ & $224,93 b$ & 221 & 1,08 & 0,05 \\
\hline $\mathrm{SM}+\mathrm{PG}+\mathrm{MO}$ & $52,08 \mathrm{a}$ & $115,90 \mathrm{a}$ & $181,12 \mathrm{a}$ & $228,79 a$ & $252,29 a$ & 248 & 1,39 & 0,05 \\
\hline
\end{tabular}

SM: silagem de milho, PG: propilenoglicol, MO: monensina; A: potencial máximo de produção de gás; Lag: tempo de colonização (h); $\mu$ : taxa de produção de gases.

Médias seguidas de letras distintas na coluna diferem entre si pelo teste $\mathrm{F}(\mathrm{P}<0,05)$. CV: 2,0\%.

A adição de monensina promoveu menor $(\mathrm{P}<0,05)$ produção de gases durante as últimas 48 horas de fermentação, quando comparado aos demais tratamentos. A produção de gases entre 6,8 e $9,8 \%$ foi menor que a apresentada pelo grupo SM, nesse período.

O menor potencial de produção de gases $(221 \mathrm{ml})$, quando comparado aos demais tratamentos, foi observado com a utilização de MO (Tab. 4).

A adição de monensina promoveu menor tempo de colonização $(1,08 \mathrm{~h})$ em relação aos tratamentos SM e SM+PG $(1,58$ e $1,49 \mathrm{~h}$, respectivamente). $\mathrm{O}$ menor tempo de colonização pode estar relacionado às mudanças da microbiota causadas pela ação da monensina. Essa possível alteração na população microbiana, também, pode ter sido responsável pelo decréscimo do potencial máximo de produção de gases.

A menor produção e o menor potencial máximo de produção de gases, observados com a adição somente da monensina, podem ser explicados pela sua ação sobre a metanogênese ruminal. Segundo Nagaraja et al. (1997), o uso de monensina promove redução nos precursores de metano, o que leva à queda na metanogênese entre 4 e 31\% (Johnson e Johnson, 1995). Além dos efeitos sobre a produção de metano, Chalupa et al. (1980) observaram menor produção de dióxido de carbono quando foram utilizados altos níveis de ionóforos.

Devido à sua atividade antimicrobiana, a monensina pode ter alterado a microbiota ruminal, o que resultou em uma população de microorganismos capazes de colonizar mais rapidamente o substrato.

Os tratamentos com PG também apresentaram maior potencial máximo de produção de gases (246ml/g MS) e menor tempo de colonização $(1,49 \mathrm{~h})$ em relação ao tratamento SM $(245 \mathrm{ml} / \mathrm{g}$ MS e 1,58h, respectivamente). O PG pode ser utilizado diretamente como substrato pelas bactérias ruminais (Clapperton e Czerkawski, 1972), o que explicaria o maior potencial máximo de produção de gases e o menor tempo de colonização. 
A correlação entre produção cumulativa de gases e degradação dos carboidratos totais foi elevada para todos os tratamentos, sendo $\mathrm{r}=94 ; 95 ; 96 \mathrm{e}$ 97\% para os tratamentos SM, SM+PG, SM+MO e $\mathrm{SM}+\mathrm{PG}+\mathrm{MO}$, respectivamente. Apesar da alta correlação, algumas diferenças foram observadas entre os resultados de degradabilidade e produção de gases. Tratamentos semelhantes quanto à degradabilidade (Tab. 1) apresentaram valores de produção cumulativa de gases diferentes (Tab. 4) $(\mathrm{P}<0,05)$.

A menor acurácia nos valores de degradabilidade pode ter ocorrido uma vez que o método é gravimétrico (Pell e Schofield, 1992). Nesse tipo de mensuração, observa-se o desaparecimento do substrato, mesmo que não contribua com a fermentação. Na técnica em que há mensuração dos gases produzidos, o que é avaliado, na realidade, é o produto da fermentação (Getachew et al., 1998; Faria et al., 2008).

Para Blümmel e Bullerdick (1997), a determinação do resíduo revela quanto substrato foi utilizado na fermentação e a mensuração dos gases revela quanto do substrato fermentado foi transformado em AGV e gases. Groot et al. (1997) ressaltaram a importância da distinção entre fermentação e degradação, principalmente para a avaliação de forragens com composição heterogênea, pois nem todo material degradado é, necessariamente, fermentado.

Na TIVSAPG, pode ocorrer produção indireta de gases, não sendo estes produzidos diretamente a partir da fermentação dos substratos. Essa produção dá-se por meio de reações de neutralização dos AGV pelo tamponante do meio de cultura (Beuvink e Spoeltra, 1992). A produção indireta pode propiciar aumento na produção e, conseqüentemente, aumento na curva cumulativa de produção de gases, superestimando a produção de gases.

Neste experimento, foram utilizados aditivos que promovem a diminuição da metanogênese. As variações observadas entre a produção cumulativa de gases e a degradabilidade podem estar relacionadas à capacidade dos aditivos em diminuir a produção de metano, sem afetar a degradação dos carboidratos totais.
Não houve diferenças significativas nos valores de $\mathrm{pH}$ entre os tratamentos, mas uma queda no $\mathrm{pH}$ do meio de cultura ao longo do período experimental. $\mathrm{O} \mathrm{pH}$ foi inversamente relacionado ao aumento da degradação dos carboidratos totais $(\mathrm{r}=-0,79, \quad \mathrm{P}<0,01$, Fig. 1). Em experimentos realizados com bovinos de leite, Oetzel et al. (1999) concluíram que o aumento na concentração de $\mathrm{AGV}$ é o fator mais importante para a queda do $\mathrm{pH}$ ruminal. Uma vez que a degradação dos carboidratos totais leva à produção de $\mathrm{AGV}$, o qual não é retirado do meio de cultura, e como a TIVSAPG é um sistema fechado, era de se esperar que houvesse o acúmulo de AGV e, por consequência, o abaixamento do $\mathrm{pH}$.

A metabolização do propilenoglicol pela microbiota ruminal resulta em AGV e, também, em ácido lático, o qual é comumente relacionado a grandes quedas no $\mathrm{pH}$ ruminal (Clapperton e Czerkawski, 1972). Já a monensina tem sido relacionada com a diminuição da produção de ácido lático, devido ao seu efeito deletério sobre as bactérias produtoras de lactato (Nagaraja et al., 1981, 1982). No entanto, não foram observados efeitos importantes desses aditivos sobre o pH do meio de fermentação. Apesar da queda apresentada no $\mathrm{pH}$, ao longo do processo de fermentação, seus valores ainda permaneceram acima de 6,5. Segundo Van Soest (1994), os microrganismos celulolíticos são muito sensíveis a quedas no $\mathrm{pH}$ ruminal. Como essa microbiota é fundamental na degradabilidade da celulose, em ambientes com pH abaixo de 6,0, a degradação da celulose torna-se mais difícil. Devido à eficiência do tampão fosfato utilizado no meio de cultura da TIVSAPG, em nenhum momento os valores de $\mathrm{pH}$ apresentaram-se abaixo de 6,5, não interferindo, assim, na degradabilidade da fibra.

É importante salientar que, in vivo, a manutenção do $\mathrm{pH}$ ruminal não é tão eficiente quanto à observada nessa técnica in vitro. As pequenas variações de $\mathrm{pH}$ observadas neste experimento, provavelmente não refletem as condições de um ruminante no período pós-prandial. 


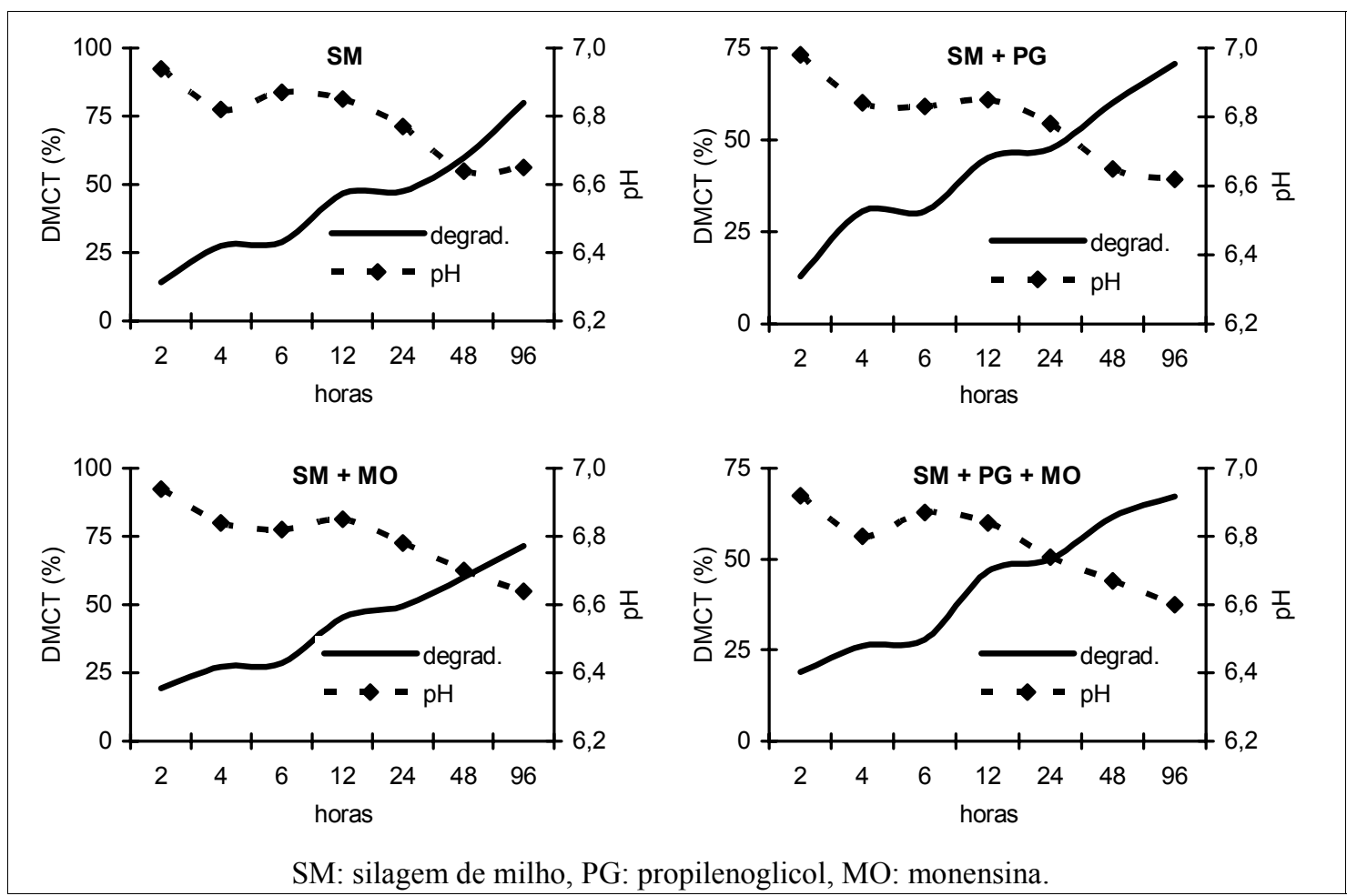

Figura 1. Degradação média dos carboidratos totais (DMCT\%) e mudanças no $\mathrm{pH}$ dos frascos de fermentação para silagem de milho associada ou não ao propilenoglicol e/ou à monensina.

\section{CONCLUSÕES}

Apesar de diminuir a produção cumulativa de gases, a utilização de monensina pode ser uma boa alternativa para se melhorarem os parâmetros da cinética de fermentação ruminal da silagem de milho. O propilenoglicol não promove alterações sobre a degradação dos carboidratos totais da silagem de milho.

\section{REFERÊNCIAS BIBLIOGRÁFICAS}

BEUVINK, J.M.W.; SPOELSTRA, S.F. Interactions between substrate, fermentation endproducts, buffering systems and gas production upon fermentation of different carbohydrates by mixed rumen microorganisms in vitro. Appl. Microbiol. Biotech., v.37, p.505-509, 1992.

BLÜMMEL, M.; BULLERDICK, P. The need to complement in vitro gas mensurements whith residue determination from in saco degradabilities to improve the prediction of voluntary intake of hays. Anim. Sci., v.64, p.7175, 1997.
CHALUPA W.; CORBETT, W.; BRETHOR, J.R. Effects of monensin and amicloral on rumen fermentation. J. Anim. Sci., v.51, p.170-179, 1980.

CLAPPERTON, J.L.; CZERKAWSKI, J.W. Metabolism of propane-1:2-diol infused into the rumen of sheep. Br. J. Nutr., v.27, p.553-560, 1972.

FARIA, B.N.; REIS, R.B.; MAURÍCIO, R.M. et al. Efeitos da adição de monensina ou propilenoglicol à polpa cítrica sobre a cinética de degradação dos carboidratos totais e da produção cumulativa de gases in vitro. Arq. Bras. Med. Vet. Zootec., v.60, p.691-697, 2008.

FRANCE, J.; DHANOA, M.S.; THEODOROU, M.K., et al. A model to interpret gas accumulation profiles with in vitro degradation of ruminant feeds. J. Theor. Biol., v.163, p.99$111,1993$.

GETACHEW, G.; BLÜMMEL, M.; MAKKAR, H.P.S., et al. In vitro gas measuring techniques for assessment of nutritional quality of feeds: a 
review. Anim. Feed Sci. Technol., v.72, p.261281, 1998.

GOODRICH，D.R.; GARRET，J.E.; GAST, M.A. et al. Influence of monensin on the performance of cattle. J. Anim. Sci., v.58, p.1484-1497, 1984.

GROOT, J.C.J.; WILLIAMS, B.A.; OOSTDAM, A.J. et al. The use of cumulative gas and volatile fatty acid production to predict in vitro fermentation kinetics of italian ryegrass leaf cell walls and contents at various time intervals. Br. J. Nutr., v.79, p.519-525, 1997.

GRUMMER, R.R.; WINKLER, J.C.; BERTICS, S.J. et al. Effect of propyleneglycol dosage during feed restriction on metabolites in blood of prepartum holstein heifers. J. Dairy Sci., v.77, p.3618-3623, 1994.

JOHNSON, K.A.; JOHNSON, D.E. Methane emissions from cattle. J. Anim. Sci., v.73, p.2483-2492, 1995.

MAURICIO, R.M.; MOULD, F.L.; DHANOA, M.S. A semi-automated in vitro gas production technique for ruminants fedstuff evaluation. Anim. Feed Sci. Technol., v.79, p.321-330, 1999.

MAURÍCIO, R.M.; PEREIRA， L.G.R.; GONÇALVES, L.C. et al. Relação entre pressão e volume para implantação da técnica in vitro semi-automática de produção de gases na avaliação de forrageiras tropicais. Arq. Bras. Med. Vet. Zootec., v.55, p.216-219, 2003.

NAGARAJA, T.G; AVERY, T.B.; BARTLEY, E.E. et al. Prevention of lactic acidosis in cattle by lasalocid or monensin. J. Anim. Sci., v.53, p.206-216, 1981.

NAGARAJA, T.G; AVERY, T.B.; BARTLEY, E.E. et al. Effect of lasalocid, monensin or thiopeptin on lactic acidosis in cattle. J. Anim. Sci., v.54, p.649-658, 1982.
NAGARAJA, T.G.; NEWBOLD, C.J.; VAN NEVEL, C.J. et al. Manipulation of ruminal fermentation In: HOBSON, N.P. (Ed). Rumen microbial ecosystem. London: Blackie, 1997. p.523-631.

NOGUEIRA, U.T.; MAURÍCIO, R.M.; GONÇALVES, L.C. Comparação de substratos com diferentes quantidades de carboidratos solúveis utilizando a técnica in vitro semiautomática de produção de gases. Arq. Bras. Med. Vet. Zootec., v.58, p. 633-641, 2006.

NUTRITIVE requirements of ruminant animal. AFRC. Nutr. Abstr. Rev., n.9, p.65-71, 1992.

OETZEL, G.R.; NORDLUND, K.F.; GARRET, E.F. Effect of ruminal $\mathrm{pH}$ and stage of lactation on ruminal lactate concentrations in dairy cows. J. Dairy Sci., v.82, suppl.1, p.38, 1999. (Abstract).

OFFICIAL methods of analisys. 13.ed. Washington: AOAC, 1980. 1015p.

PELL A.N.; SCHOFIELD, P. Computerized monitoring of gas production to measure forage digestion in vitro. J. Dairy Sci., v.76, p.10631073, 1992.

SCHELLING, G.T. Monensin mode of action in the rumen. J. Anim. Sci., v.58, p.1518-1527, 1984.

SIMPSON, M.E. Effects of certain antibiotics on in vitro cellulose digestibility and volatile fatty acid production by ruminal microorganisms. $J$. Anim. Sci., v.47, Suppl.1, p. 429, 1978.(Abstract).

SISTEMA de análises estatísticas - SAEG. Viçosa: UFV, 1997. 52p.

VAN SOEST, P.J. Nutritional ecology of ruminant. 3.ed. Ithaca: Cornell University, 1994. 476p. 